\title{
中国北方5种栋属树木多度分布及其对未来气候变 化的响应
}

\author{
${\text { 张雪皎 }{ }^{1} \text { 高贤明 }{ }^{2} \text { 吉成均 }{ }^{1} \text { 康慕谊 }{ }^{3,4} \text { 王仁卿 }}^{5}$ 岳 明 ${ }^{6}$ 张 峰 ${ }^{7}$ 唐志尧 $1^{*}$
}

${ }^{1}$ 北京大学城市与环境学院, 北京大学生态研究中心, 地表过程分析与模拟教育部重点实验室, 北京 $100871 ;{ }^{2}$ 中国科学院植物研究所植被与环境变化 国家重点实验室, 北京 $100093 ;{ }^{3}$ 北京师范大学地表过程与资源生态国家重点实验室, 北京 $100875 ;{ }^{4}$ 北京师范大学地理科学学部自然资源学院, 北京 $100875 ;{ }^{5}$ 山东大学生命科学学院, 济南 $250100 ;{ }^{6}$ 西北大学西部资源生物与现代生物技术教育部重点实验室, 西安 $710069 ;{ }^{7}$ 山西大学黄土高原研究 所, 太原 030006

摘 要 植物分布与气候之间的关系是预估未来气候变化对生态系统影响的实现基础。以往的物种分布模型通常以物种的分 布区或者分布点的物种存在数据作为物种分布的响应变量。相较于物种存在数据, 多度反映了一个物种占用资源并把资源分 配给个体的能力, 更能衡量物种对区域生态系统的影响。该研究通过野外调查获取了华北及周边地区 1045 个样方的栎属树木 多度，利用广义线性模型、广义加性模型和随机森林模型模拟栓皮栋(Quercus variabilis)、麻栎(Q. acutissima)、槲栋(Q.aliena)、 锐齿槲柇(Q. aliena var. acuteserrata)和蒙古柇(Q. mongolica) 5 个树种多度的地理分布及未来 2 个不同时期(2050年和 2070 年)的 潜在分布。结果表明: 随机森林模型对 5 个栎属树种的多度的拟合结果要优于广义线性模型和广义加性模型; 典型浓度路径 (RCP) 8.5 下的 5 个栎属树种在未来两个时期的多度变化幅度都要大于RCP 2.6 下的变化, 在超过一半面积的区域中麻栎、桷 栎、锐齿葪栎和蒙古柇的多度减少, 其中内蒙古东北部和黑龙江北部地区是 5 种栎属植物多度减少的集中分布地区。未来气 候变化背景下, 需要加强对这几个区域的监测与物种保护。

关键词 广义线性模型; 广义加性模型; 随机森林模型; 物种分布模型

张雪皎, 高贤明, 吉成均, 康慕谊, 王仁卿, 岳明, 张峰, 唐志尧 (2019). 中国北方 5 种栎属树木多度分布及其对未来气候变化的响应. 植物生态学报, 43, 774-782. DOI: $10.17521 /$ cjpe.2018.0249

\section{Response of abundance distribution of five species of Quercus to climate change in northern China}

ZHANG Xue-Jiao ${ }^{1}$, GAO Xian-Ming ${ }^{2}$, JI Cheng-Jun ${ }^{1}$, KANG Mu-Yi ${ }^{3,4}$, WANG Ren-Qing ${ }^{5}$, YUE Ming ${ }^{6}$, ZHANG Feng $^{7}$, and TANG Zhi-Yao ${ }^{1 *}$

${ }^{1}$ Institute of Ecology, College of Urban and Environmental Sciences, Laboratory for Earth Surface Processes of the Ministry of Education, Peking University, Beijing 100871, China; ${ }^{2}$ State Key Laboratory of Vegetation and Environmental Change, Institute of Botany, Chinese Academy of Sciences, Beijing 100093, China; ${ }^{3}$ State Key Laboratory of Earth Surface Processes and Resource Ecology, Beijing Normal University, Beijing 100875, China; ${ }^{4}$ College of Resources Science \& Technology, Faculty of Geographical Science, Beijing Normal University, Beijing 100875, China; ${ }^{5}$ School of Life Sciences, Shandong University, Jinan 250100, China; ${ }^{6}$ Key Laboratory of Resource Biology and Biotechnology in Western China, Ministry of Education, Northwest University, Xi'an 710069, China; and ${ }^{7}$ Institute of Loess Plateau, Shanxi University, Taiyuan 030006, China

\section{Abstract}

Aims To develop a statistically appropriate species distribution model for the abundance of five species from Quercus in the northern China, and to predict the change of abundance under climate change.

Methods We surveyed abundance data of five Quercus species from 1045 plots in the northern China, and then fit the abundance with climatic variables using random forest model (RF). We then predict the abundance of these five Quercus species in 2050 and 2070 under Representation Concentration Pathways (RCP) 2.6 and 8.5.

Important findings The change magnitudes of abundance for all 5 species under RCP 8.5 were larger than under RCP 2.6. Except for Quercus variabilis, abundances of other four species declined under climate change to 2050 and 2070 in more than half of the current distribution areas. Moreover, the northeastern part of Nei Mongol and the northern part of Heilongjiang will be the hotspots of decrease of abundance. Therefore, it is necessary to strengthen the monitoring and species protection in the areas mentioned above with the increasing threaten of climate change.

Key words generalized linear model; generalized additive model; random forest model; species distribution model

收稿日期Received: 2018-10-11 接受日期Accepted: 2019-01-30

基金项目：国家科技基础性工作专项(2011FY110300和2015FY210200)。Supported by the National Basic Work of Science and Technology of China (2011FY110300 and 2015FY210200).

* 通信作者Corresponding author (zytang@urban.pku.edu.cn) 
Zhang XJ, Gao XM, Ji CJ, Kang MY, Wang RQ, Yue M, Zhang F, Tang ZY (2019). Response of abundance distribution of five species of Quercus to climate change in northern China. Chinese Journal of Plant Ecology, 43, 774-782. DOI: 10.17521/cjpe.2018.0249

植物分布-气候关系是生态学和地理学研究的 热点问题，也是预估未来气候变化对生态系统影响 的实现基础(周广胜和张新时, 1996; Chen et al., 2011; Bellard et al., 2012)。物种分布模型或生态位 模型通过统计方法描述物种对气候的响应关系，判 断物种的潜在分布区, 并预测气候变化情景下物种 的未来分布区(Franklin et al., 2009)。传统的物种分 布模型通常以物种的分布区或者分布点的物种存在 数据(即出现与否)作为物种分布的响应变量(李国庆 等, 2013)。然而, 植物有多种方式响应气候变化, 除 了改变其分布区以外, 还会改变其生长物候、生理 活动强度, 或者是多度(Spano et al., 1999; Sturm et al., 2001; Parmesan, 2006)。相较于物种存在数据, 多度反映了一个物种占用资源并把资源分配给个体 的能力, 更能衡量物种对区域生态系统的影响 (Kaspari, 2001; Syphard \& Franklin, 2009; Ehrlén \& Morris, 2015)。

近100年以来, 华北及周边地区经历了高于全 国平均水平的升温趋势, 年平均气温增温速率达到 $1.1{ }^{\circ} \mathrm{C} \cdot 100 \mathrm{a}^{-1}$ (王昭武等, 1998)。栎属(Quercus) 是壳 斗科中最大的属, 分布极为广泛, 在欧亚从印度尼 西亚的爪哇岛直到北欧 $62^{\circ} \mathrm{N}$ 的斯堪迪纳亚半岛和 $52^{\circ} \mathrm{N}$ 的鄂霍茨克海域附近(周浙昆, 1992)。栎属树 种是温带落叶阔叶林的优势成分, 分布于全国各地 (彭炎松等, 2007), 也是我国华北及周边地区常见的 森林优势种。在气候变化背景下, 准确预测栎属植 物的未来分布, 将促进华北地区的植被动态、植被 管理以及脆弱生态系统的修复。本文通过大量野外 调查, 获取了华北地区常见栎属树种的多度, 利用 物种分布模型拟合其多度分布并预测其在未来气候 变化下的多度分布。

\section{1 材料和方法}

\section{1 数据收集}

研究以野外样方调查所获取的栎属植物多度作 为响应变量。在华北及周边地区共调查 1045 个柇林 的样方(图1), 每个样方面积为600-1000 $\mathrm{m}^{2}$ 。在每 个样方, 调查乔木层所有胸径 $\geqslant 3 \mathrm{~cm}$ 的树木, 记录 其胸径、树高, 共记录到栎属树木 5 种: 栓皮柇 $(Q$. variabilis $)$ 、槲栋 $(Q$. aliena $) 、$ 锐齿葪栋 $(Q$. aliena var. acuteserrata) 、蒙古栋 $(Q$. mongolica $) 、$ 麻栋 $(Q$. acutissima)。为统一单位和方便计算, 将样方中的 各种栎属植物的密度统一折算为 $1000 \mathrm{~m}^{2}$ 中的个 体数量。这些样方的乔木层平均物种数为 7 种. $1000 \mathrm{~m}^{-2}$, 平均树木密度为 95 棵. $1000 \mathrm{~m}^{-2}$ (表1)。

研究使用的气候数据从世界气候数据库

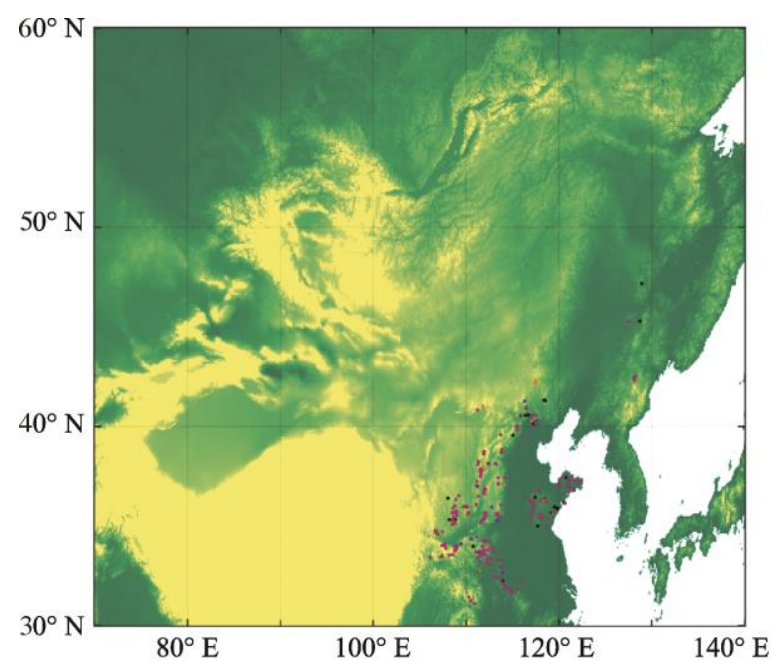

栓皮柇 $Q$. variabilis 麻标 $Q$. acutissima 檞栋 $Q$. aliena 锐齿櫓标 $Q$. alienavar var. acuteserrata 辽东栋 $Q$. wutaishanica

图1 栎属5种植物在中国北部的样方分布图。

Fig. 1 Plots distribution of five species of Quercus in northern China.

表1 中国北方5种常见柇属树木样方调查统计

Table 1 Survey statistics of plots of five species in Quercus in the northern China

\begin{tabular}{lcccc} 
物种 & $\begin{array}{c}\text { 样方数 } \\
\text { Plot number }\end{array}$ & $\begin{array}{c}\text { 平均密度 } \\
\text { Mean density } \\
\left(\text { Ind. } 1000 \mathrm{~m}^{-2}\right)\end{array}$ & $\begin{array}{c}\text { 样方的气温范围 } \\
\text { Air temperature range } \\
\text { of plots }\left({ }^{\circ} \mathrm{C}\right)\end{array}$ & $\begin{array}{c}\text { 样方的降水范围 } \\
\text { Precipitation range of } \\
\text { plots }(\mathrm{mm})\end{array}$ \\
\hline 栓皮栋 Q. variabilis & 304 & $89 \pm 101$ & $6.8-15.9$ & $529-1431$ \\
麻栋 Q. acutissima & 195 & $39 \pm 55$ & $4.1-15.8$ & $451-1614$ \\
葪栋 Q. aliena & 188 & $22 \pm 43$ & $3.6-16.0$ & $335-1775$ \\
锐齿葪栋 Q. aliena & 147 & $37 \pm 34$ & $1.4-11.6$ & $337-1502$ \\
蒙古栋 Q. mongolica & 492 & $55 \pm 68$ & $0.1-14.4$ & $335-1006$ \\
\hline
\end{tabular}


(http://www.worldclim.org/, Hijmans et al., 2005)提 取, 该数据库的Version 1.4收集了1960-2000年世界 各地气象站记录的气象信息, 插值形成分辨率为 $30^{\prime \prime} \times 30^{\prime \prime}$ (约 $1 \mathrm{~km} \times 1 \mathrm{~km}$ ) 的全球气候栅格数据, 并 基于每月气温和每月降水记录, 计算得到 19 个生物 气候因子, 分别为年平均气温、气温日较差、等温 性、气温季节性、最热月最高气温、最冷月最低气 温、气温年较差、最湿季平均气温、最干季平均气 温、最热季平均气温、最冷季平均气温、年降水量、 最湿月降水量、最干月降水量、降水季节性、最湿 季降水量、最干季降水量、最热季降水量和最冷季 降水量。

未来气候变化情景选取中国气象局北京气候中 心(BCC-CSM 1.1)提供的大气环流模型结果, 该结 果预测了 4 种典型浓度路径(RCP) 以表征不同温室 气体排放情境，即RCP 2.6、RCP 4.5、RCP 6.0、RCP 8.5 。本研究中采用了该气候模型的两个极端排放情 景, 即RCP 2.6 和RCP 8.5 的气候模拟结果。 RCP 2.6 情景设定温室气体的综合排放水平在未来先上升后 下降, 辐射强度为 4 种情景中最低; RCP 8.5 情景设 定没有减排措施, 温室气体的排放水平在未来持续 增加, 辐射强度为 4 种情景中最高, 气候变化状况最 显著(Riahi et al., 2011; van Vuuren et al., 2011; Kharin et al., 2013)。以上气候数据按照研究区裁剪。 在预测时用 $1: 100$ 万植被分布图(中国科学院中国植 被图编辑委员会, 2007)对气候图层进行掩膜裁剪, 提取其中森林和灌丛植被类型，。

\section{2 模型选择}

本文选取了广义线性模型 (GLM; Nelder \& Wedderburn, 1972)、广义加性模型(GAM; Hastie \& Tibshirani，1986)和随机森林模型 (RF; Cutler \& Breiman, 2004)来模拟物种多度与环境的关系。这几 种模型已被成功用于树木和灌丛的分布模型 (Thuiller, 2003; Thuiller et al., 2003; Dubuis et al., 2011; Wang et al., 2017)。

GLM 具有较强的预测物种分布的能力(Austin \& Meyers, 1996; Brito et al., 1999); GAM适用于响 应变量与解释变量之间的非线性关系, 本研究中的 样方并未覆盖物种完整的生态位, 在事先没有关于 响应变量与解释变量关系的明确假设时, GAM可以 很好地探索这种关系(Wang et al., 2017)。RF是基于 决策树的集成算法, 不用对函数形式进行事先假定,
避免了假设误差, 不需要顾虑一般回归分析面临的 多元共线性的问题, 不用做变量选择(Cutler \& Breiman, 2004), 被誉为当前最好的算法之一 (Iverson et al., 2008), 在物种分布模拟中得到了广 泛应用(Cutler et al., 2007)。

GLM和GAM中, 增加自变量数目可以提高模 型解释因变量的能力, 但具有多重共线性的多个自 变量应用会导致模型过度拟合(Sainani, 2013)。为了 避免过度拟合风险, 本研究通过计算19个气候因子 的Pearson相关系数, 用单个因子对物种分别进行一 次预模拟。基于模型的赤池信息量准则(AIC)值和各 因子之间的相关性，选取拟合度高且共线性小的因 子 $(|r|<0.7)$ (Yan et al., 2017), 同时检测所选因子二 次项是否显著。最终GLM包含所选因子的一次项和 检验显著的二次项, GAM包含与线性模型相同的一 次项因子。对于最终建立的GLM和GAM, 栓皮柇的 预测模型包含了等温性、最热月最高气温、最冷月 最低气温、降水季节性和最湿季降水量 5 个因子; 麻 栎的预测模型包含了等温性、最冷月最低气温、气 温年较差、最湿季平均气温、最干月降水量和最热 季降水量6个变量; 槲栋的预测模型包含了等温性、 最湿季平均气温、最干季平均气温、年降水量和最 湿月降水量 5 个变量; 锐齿檞柇的预测模型包含等 温性、最冷月最低气温、最湿季平均气温、最干月 降水量和降水季节性等 5 个变量; 蒙古栎的预测模 型包含了年平均气温、等温性、气温季节性、最湿 季平均气温、年降水量、最湿月降水量和最热季降 水量等7个变量。由于RF中不存在共线性问题, 使用 19 个气候因子作为自变量, 二叉树变量和森林数目 选择使模型残差最小的值。

模型准确度用模型预测值与实际观测值之间的 Pearson相关系数来表征(Young \& Carr, 2015)。为验 证模型的模拟质量, 用 $k$ 折交叉验证来校验模型, 将 数据集等分为 $k$ 份, 每个子集均做一次测试集, 其余 的作为训练集, 交叉验证重复 $k$ 次。每次选择一个子 集作为测试集, 并将 $k$ 次的交叉验证平均值作为准 确率, 这样, 所有的样本都可以被作为训练集和测 试集, 每个样本都被验证一次, 可以充分利用已有 数据集对算法效果进行测试, 本研究采用 10 折交叉 验证, 最终准确度为 100 次 10 折交叉验证结果 (Picard \& Denniscook, 1984)。在5种栎属树木中, GLM对栓皮柇的拟合结果最佳 $(r=0.42)$; RF对栓皮

www.plant-ecology.com 
栋、锐齿檞栋、麻栎的拟合结果较好 $(r$ 值分别为 0.61 、 $0.63 、 0.53)$, 对䚞柇的拟合结果较差 $(r=0.36)$ 。总体 而言, RF对 5 种树木的拟合结果都要优于 GAM和 GLM (图2), 因此在后续结果中只采用随机森林进 行基准气候与未来气候情景下物种多度的预测。预 测结果用 100 次 10 折交叉验证建立起来的模型预测 的平均值来表示。

\section{2 结果}

\subsection{5 种栋属树木的潜在多度分布}

现代气候条件下，随机森林对不同物种多度的 模拟结果表明，栓皮柇在陕西省中部、山西省北部、 河北省东北部、北京市北部和黑龙江省北部多度较 大；麻栎多度较大的地区大致处于陕西省中部、山 西省北部地区; 槲栋在陕西省中部、山西省北部分 布数量较多; 锐齿葪柇在黑龙江省东部、吉林省西 南部、辽宁省东部分布数量较多; 蒙古栎在内蒙古 自治区东北部、黑龙江省北部及两省区交界处及陕 西省北部分布较多(图3)。

\section{2 未来气候变化背景下 5 种栋属树木的多度变化}

与现代气候条件下的多度分布相比，2050年 RCP 2.6 下，栓皮柇、麻栋、葪栎、锐齿檞栎和蒙古 标的多度变化分别为平均 $1.17 \% 、-6.55 \% 、-4.84 \%$ 、 $-8.78 \%$ 和 $-0.77 \%$, 除栓皮栋外，其他 4 个物种的平 均多度都减小。其中, 栓皮柇、檞柇和锐齿檞係的 变化不显著, 而麻栎和蒙古係的变化显著。对栓皮 柇、麻栋、䚞栎、锐齿䚞柇和蒙古栎来说, 多度减 少区域的面积分别占到总分布区域的 $47.1 \%$ 、 $65.6 \% 、 59.8 \% 、 66.3 \%$ 和 $55.2 \%$ ，也就是说，除了栓 皮柇外, 其他 4 个种在该气候情景下有超过一半区 域的多度是减少的。 RCP 8.5 下，栓皮栋、麻栎、檞 栎、锐齿檞柇和蒙古楿的多度变化分别为平均 $2.9 \%$ 、 $-2.81 \% 、-2.19 \% 、-16.23 \%$ 和 $6.69 \%$, 栓皮柇和蒙古 柇的多度增加, 麻栎、葪柇和锐齿葪柇的多度减少, 其中, 麻栎多度减少显著, 其他 4 个种的变化都不显 著。对于栓皮栋、麻栎、檞栎、锐齿檞柇和蒙古栎 来说，多度减少区域的面积分别占到总分布区域的 $45.7 \% 、 60.4 \% 、 59.4 \% 、 77.3 \%$ 和 $43.9 \%$ ，麻栎、桷
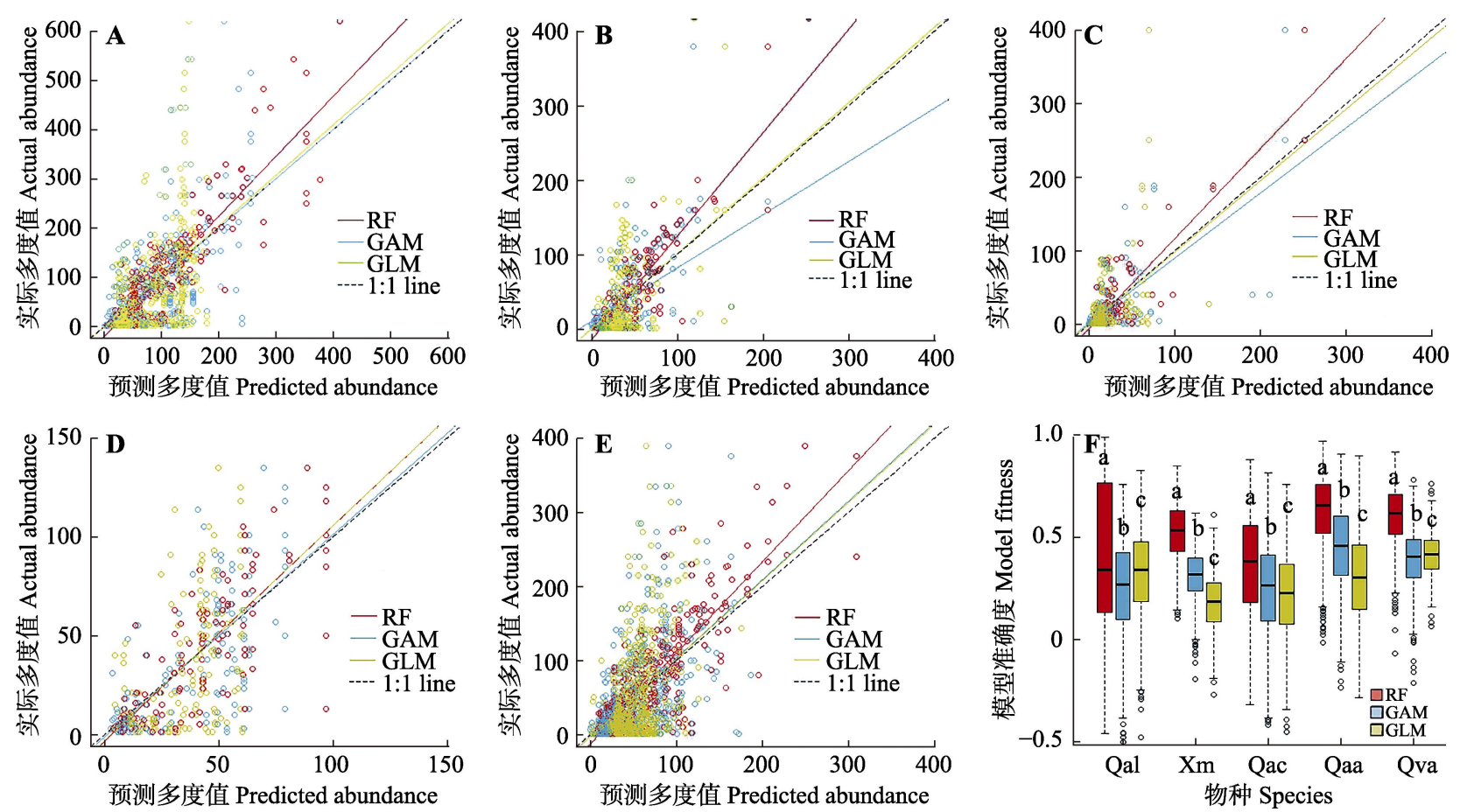

图2 现有气候条件下随机森林模型 $(\mathrm{RF})$ 、广义加性模型 $(\mathrm{GAM})$ 和广义线性模型(GLM)对预测的栎属5种植物的潜在分布多度

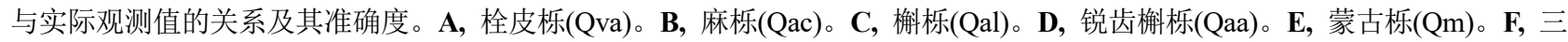
个模型的准确度比较, 不同小写字母表示模型间差异显著 $(p<0.05)$ 。

Fig. 2 Relationship between observed and predicted species abundances for five species in Quercus with random forest model (RF), generalized additive model (GAM), generalized linear model (GLM) and comparison of the accuracy of five species based on general linear model and random forest. A, Q. variabilis (Qva). B, Q. acutissima (Qac). C, Q. aliena (Qal). D, Q. aliena var. acuteserrata (Qaa). E, Q. mongolica (Qm). F, Model fitness of the three models, differed lowercase letters indicated significant differences between the models $(p<0.05)$. 
栋和锐齿槲栋有超过一半区域的多度是减少的，锐 齿檞栎多度减少面积的比例达到 $70 \%$ 以上(图4)。 2050年，栓皮栋、麻栋、葪栋、锐齿葪栋和蒙古栎
的多度在RCP 2.6 和RCP 8.5 下多度都集中减少的区 域包括陕西省中部、内蒙古自治区东北部和黑龙江 省北部区域。此外，锐齿槲柇的多度在这两种气候
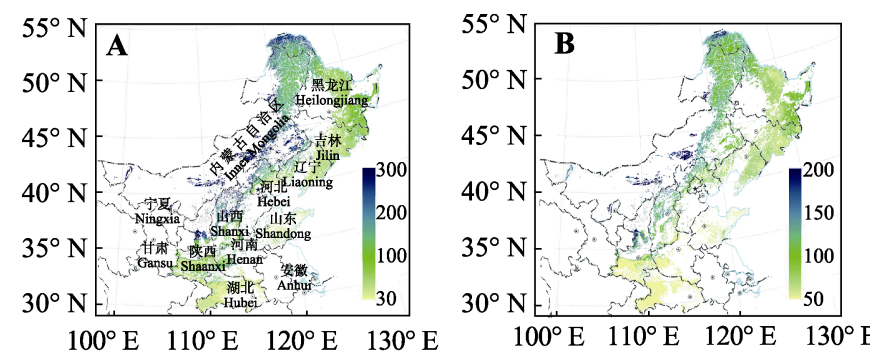

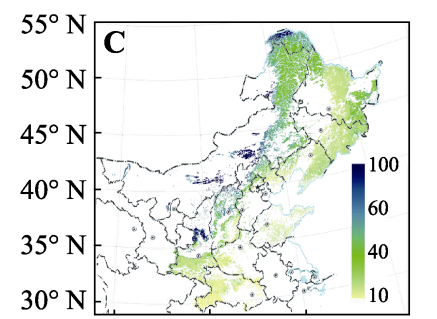

$100^{\circ} \mathrm{E} \quad 110^{\circ} \mathrm{E} \quad 120^{\circ} \mathrm{E} \quad 130^{\circ} \mathrm{E}$
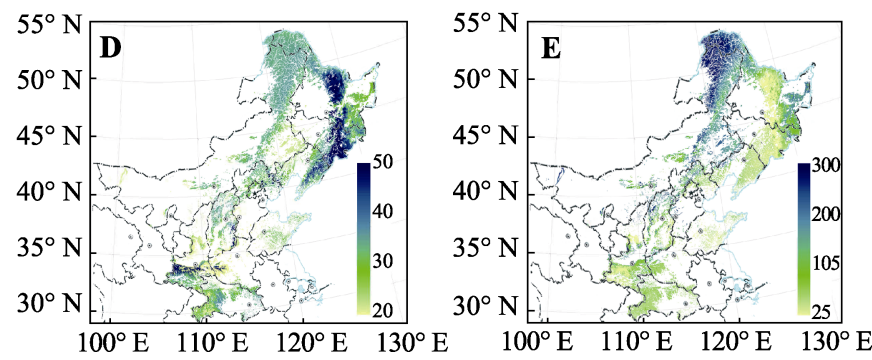

图3 中国北方5种树种在基准气候(1960-1990年)的多度(棵·1000 $\mathrm{m}^{-2}$ )潜在分布。A, 栓皮柇。 B, 麻栋。C , 檞柇。 D, 锐齿檞 栋。E, 蒙古栎。

Fig. 3 Species abundance (number $1000 \mathrm{~m}^{-2}$ ) distribution maps of the North China for five species produced by random forest model, based on bioclimatic variables at year 1960-1990. A, Quercus variabilis. B, Q. acutissima. C, Q. aliena. D, Q. aliena var. acuteserrata. E, Q. mongolica.
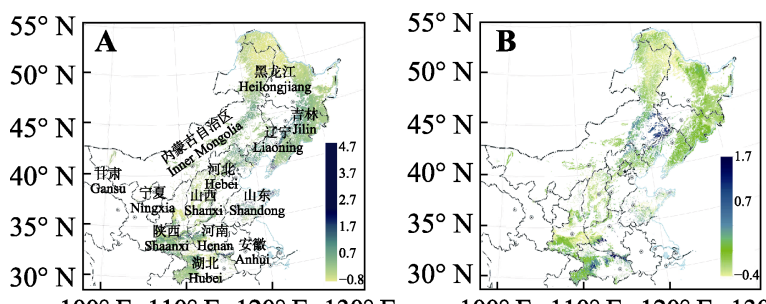

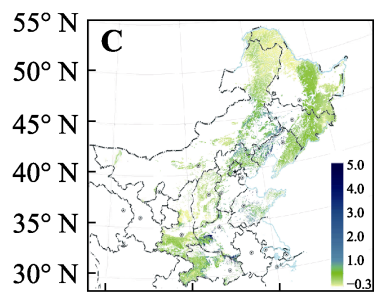

$100^{\circ} \mathrm{E} 110^{\circ} \mathrm{E} 120^{\circ} \mathrm{E} 130^{\circ} \mathrm{E}$

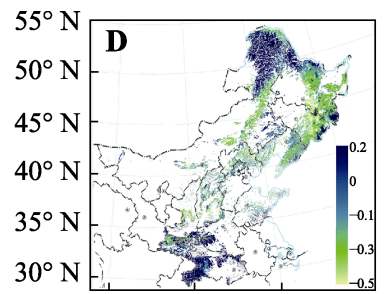

$100^{\circ} \mathrm{E} 110^{\circ} \mathrm{E} 120^{\circ} \mathrm{E} 130^{\circ} \mathrm{E}$
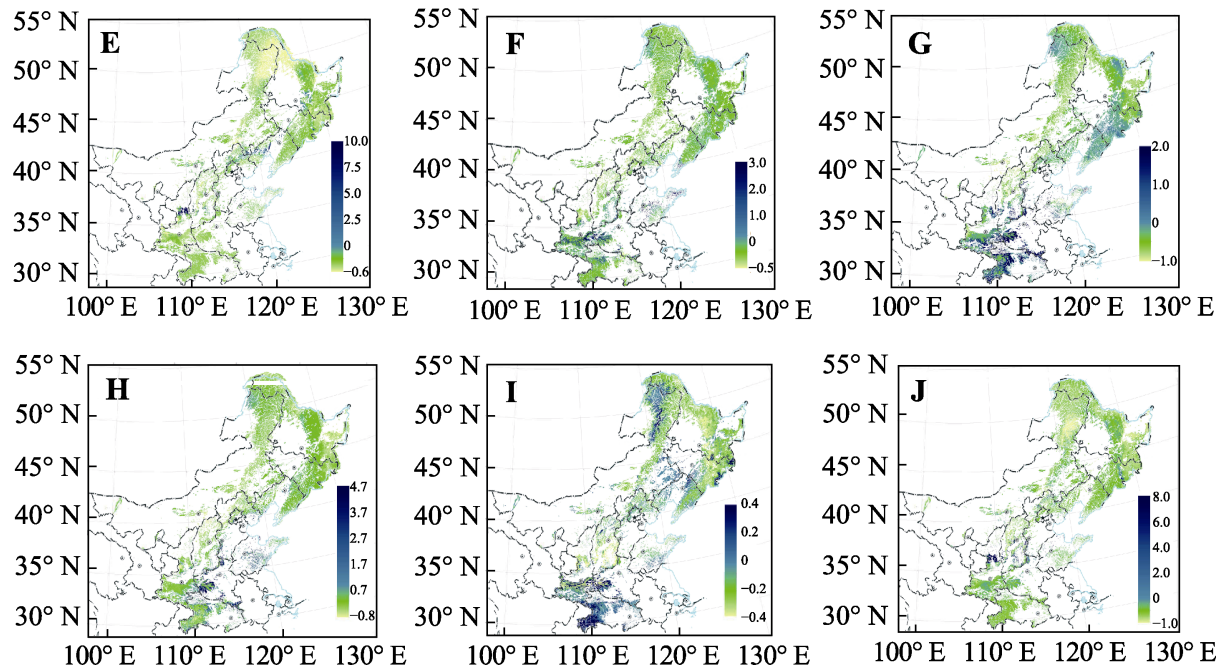

图4 中国北方5种树种在2050年时期RCP 2.6 (A-E)和RCP 8.5 (F-J)下预测多度值与现在基准(1960-1990年)的差值变化率分 布。A, F, 栓皮栋。 B, G, 麻栎。C, H, 檞栎。D, I, 锐齿檞栋。 E, J, 蒙古栎。

Fig. 4 Future distribution maps of rate of abundance change in the North China for five species produced by random forest model, based on bioclimatic variables under RCP 2.6 dispersal scenario (A-E) and RCP 8.5 dispersal scenario (F-J) in year 2050. A, F, Quercus variabilis. B, G, Q. acutissima. C, H, Q. aliena. D, I, Q. aliena var. acuteserrata. E, J, Q. mongolica. 
情景下都在吉林省东南部、辽宁省东部和陕西省南 部减少。栓皮栋、葪柇和蒙古柇在RCP 2.6 和RCP 8.5 下多度都增多的地区有黑龙江省中部和吉林省东部, 此外，麻栎的多度在吉林省东部增加。

与现代气候条件下的多度分布相比, 2070年 RCP 2.6下，栓皮栋、麻栋、檞栋、锐齿槲柇和蒙古 栋的多度变化分别为平均 $1.46 \% 、 0.07 \% 、-0.48 \%$ 、 $-8.45 \%$ 和 $2.05 \%$, 栓皮栋、麻栎和蒙古柇的多度是增 加的, 槲柇和锐齿槲係的多度是减少的, 5 个物种的 增加(减少)都不显著。对于这 5 个物种, 多度减少区 域的面积分别占到总分布区域的 $48.6 \%$ 、 $54.4 \%$ 、 $56.4 \% 、 64.4 \%$ 和 $52.4 \%$, 麻栋、葪栋、锐齿葪栋和蒙 古栎都有超过一半区域的多度是减少的。 RCP 8.5 下，栓皮栎、麻栋、槲栋、锐齿葪栋和蒙古柇的多 度变化分别为平均 $-5.45 \% 、-7.99 \% 、-4.2 \% 、-12.87 \%$ 和 $-2.05 \%, 5$ 个物种的多度都是减少的, 其中锐齿葪 栎多度的减少不显著, 而其他 4 个种多度的减少都 是显著的。对于这 5 个物种, 多度减少区域的面积分 别占到总分布区域的 $61.6 \% 、 62.0 \% 、 61.3 \% 、 74.4 \%$ 和 $52.4 \%$, 也就是说 5 个物种都有一半以上区域的多
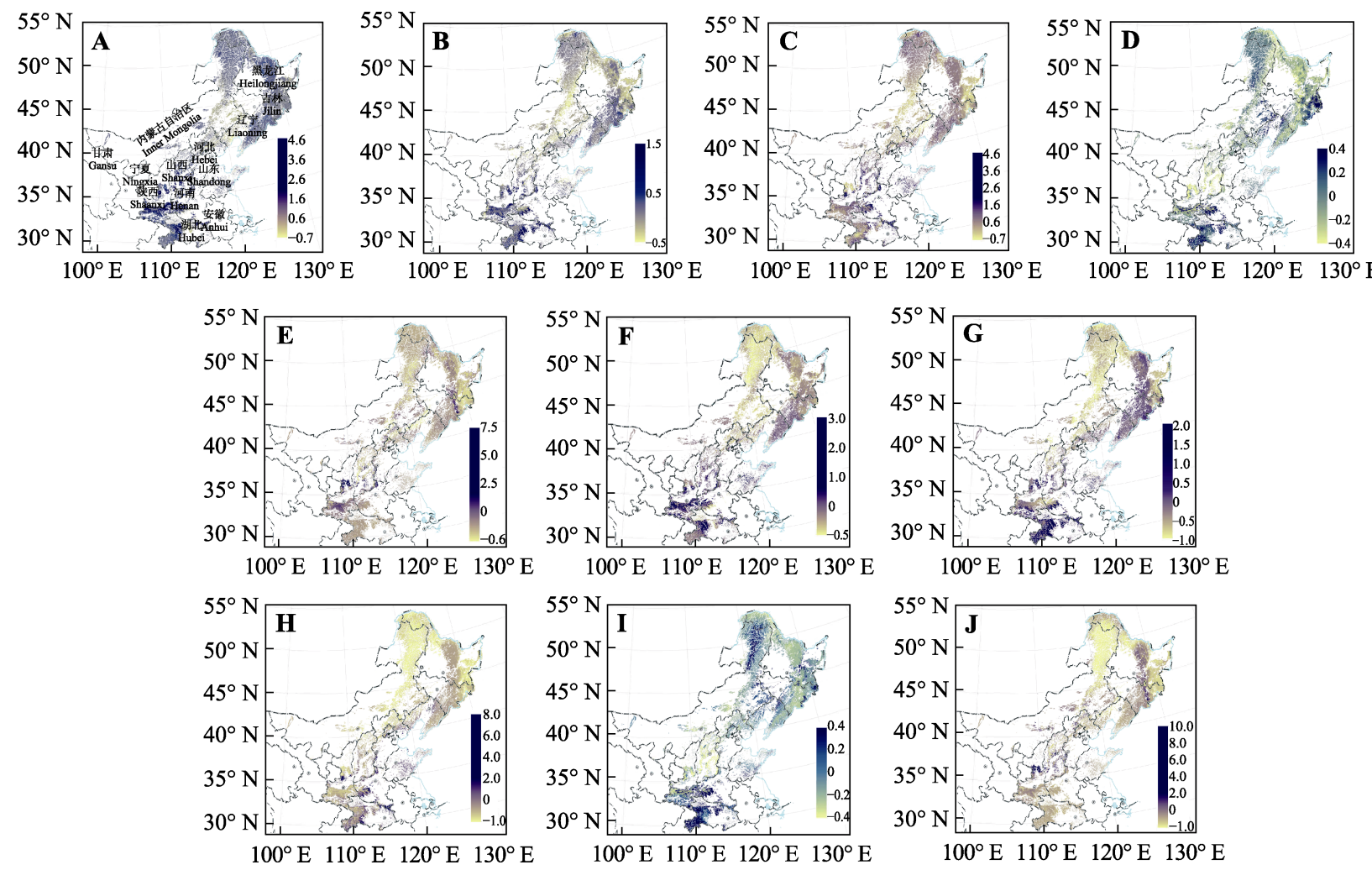

图5 中国北方5种树种在2070年时期RCP 2.6 (A-E)和RCP 8.5 (F-J)下预测多度值与现在基准(1960-1990年)的差值变化率分 布。A, F, 栓皮栋。 B, G, 麻栋。C, H, 槲栋。D, I, 锐齿槲栋。 E, J, 蒙古栋。

Fig. 5 Future distribution maps of rate of abundance change in the North China for five species produced by Random Forest models, based on bioclimatic variables under RCP 2.6 dispersal scenario (A-E) and RCP 8.5 dispersal scenario (F-J) in year 2070. A, F, Quercus variabilis. B, G, Q. acutissima. C, H, Q. aliena. D, I, Q. aliena var. acuteserrata. E, J, Q. mongolica.

DOI: $10.17521 /$ cjpe. 2018.0249 


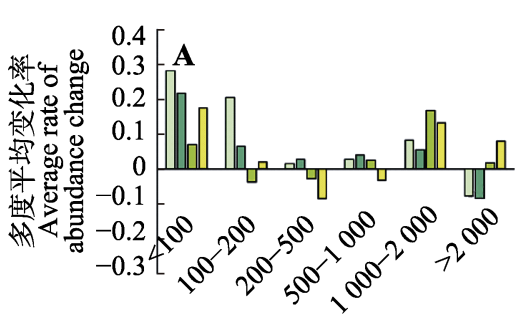

海拔 Altitude (m)

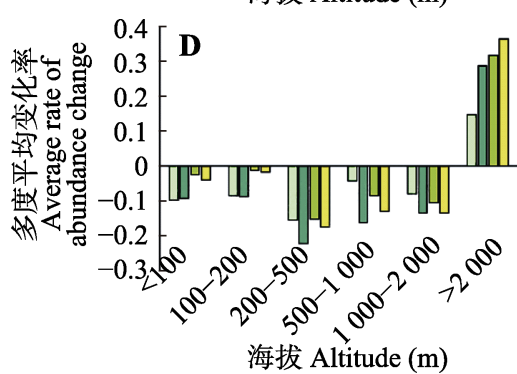

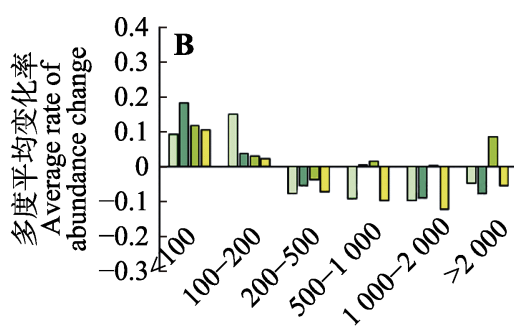

海拔 Altitude (m)

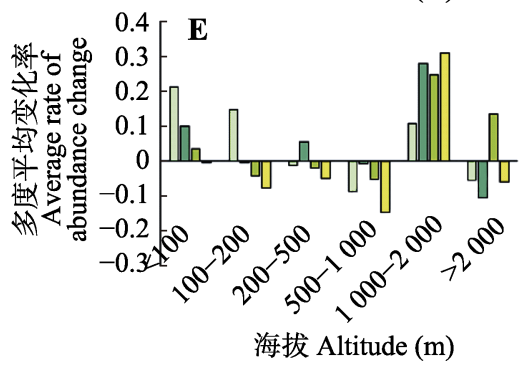

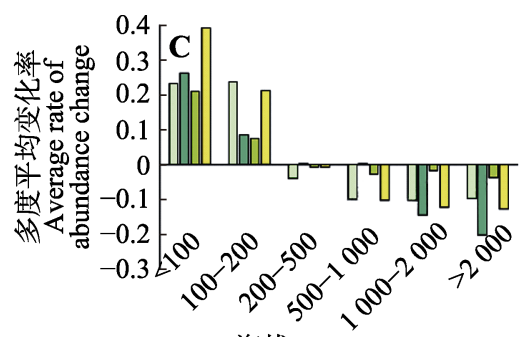

海拔 Altitude (m)

口 2050年RCP 2.6 Year 2050, RCP 2.6

口 2050年RCP 8.5 Year 2050, RCP 8.5

口 2070年RCP 2.6 Year 2070, RCP 2.6

口 2070年RCP 8.5 Year 2070, RCP 8.5

图6 中国北方不同时期及排放情景下 5 种栎属植物在各海拔区间的多度平均变化率。 $\mathbf{A}$, 栓皮栋。 $\mathbf{B}$, 麻栎。 C, 槲栎。 D, 锐 齿葪栋。E，蒙古栋。

Fig. 6 Elevation distribution of abundance change ratio of different periods under different scenario in North China. A, Quercus variabilis. B, Q. acutissima. C, Q. aliena. D, Q. aliena var. acuteserrata. E, Q. mongolica.

$1.9{ }^{\circ} \mathrm{C}$ 到 $4.4{ }^{\circ} \mathrm{C}$, 升温幅度最大的地方在研究区域 东北部, 尤其是黑龙江省北部和内蒙古自治区东部, 升温幅度从 $1.9{ }^{\circ} \mathrm{C}$ 到 $5{ }^{\circ} \mathrm{C}$ 。在降水变化方面, 大部分 地区未来向着更湿润的方向发展, 降水增幅最大出 现在内蒙古自治区西部, 最暖季降水的增幅平均小 于年降水量的增幅。RCP 8.5 下的升温幅度与降水增 幅都要高于RCP 2.6 下。在2050年两个情景的预测中, 研究区域RCP 8.5 下的栓皮柇、麻柇、䚞栎和蒙古栎 多度平均增加值都要大于 RCP 2.6, 而锐齿檞栎的 多度在RCP 8.5 下减少值大于RCP 2.6下。到2070年, 栓皮栎、麻栎、葪柇、锐齿檞栎和蒙古柇在RCP 8.5 下多度增加值都小于RCP 2.6下的值。也就是说, 5 个物种在未来两个时期中RCP 8.5 下多度增加(减少) 的值都要大于RCP 2.6 下的值。栓皮柇、麻栎和䚞柇 的多度在低海拔地区增加值高于高海拔地区，而 锐齿葪栎和蒙古柇的多度则是在高海拔地区增加 较多。

在2050年完全没有减排措施的RCP 8.5 下，栓 皮栋、麻栎、檞栋和蒙古栎的多度平均增加值都要 大于 RCP 2.6下, 可能跟该情境下温度的季节性变 异和极端低温事件大大减少有关 $(X u ＆ \mathrm{Xu}, 2012$; Kharin et al., 2013)。在2070年比较切合实际的低浓 度排放情景下，栓皮柇、麻栎、蒙古栎的多度平均 值也是增加的, 可能与气候变暖导致区域最高气温 和最低气温的升高, 使生长季延长有关(Loehle \&
LeBlanc，1996)。对于许多北方阔叶树，气温上升5 ${ }^{\circ} \mathrm{C}$ 依然在其适应生长的范围之内(郝占庆等, 2001), 而在增温背景下, 潜在蒸散会增加, 对水资源的消 耗将更加显著(Rong \& Tu, 2001)。麻栎、葪栎、锐 齿檞栎和蒙古柇的多度在未来预测中, 有超过一半 面积的区域都处于减少状态。2050年和2070年，栓 皮栋、麻栎、檞栎、锐齿檞柇和蒙古柇的多度都在 内蒙古自治区东北部、黑龙江省北部地区减少，可 能是由于内蒙古自治区东北部、黑龙江省北部在未 来气候情景中降水量增幅较小, 5 个物种的多度在该 地区集中减少可能是由于降水量减少或者降水量增 加不足以平衡气温升高和潜在蒸散的加大 (Domonkos，2001)，此外，5个物种的多度在2050年 都在陕西省中部地区减少，可能是因为陕西省中部 地区在2050年RCP 2.6 下出现年降水量减少。

本研究以物种的绝对多度作为因变量, 多度相 较于物种存在数据, 反映了一个物种占用资源并把 资源分配给个体的能力, 更能衡量物种对区域生态 系统的影响在区域和洲际尺度。但本研究也存在以 下不足。气候因素是控制物种分布的主要环境因子, 随着空间尺度的减小, 其他的一些因素, 如地形、土 壤等将会对物种分布产生重大影响(曹铭昌等, 2005)。关于草场草本植物的研究已经发现, 相对于 只有气候因子的多度解释模型, 地形因子的加入可 以提升至少 $10 \%$ 的模型解释率(Wang et al., 2017)。 
此外, 物种的扩散还有可能受到地形阻碍, 本研究 也没有区分个体的大小及其他个体差异, 研究设定 多度与气候的关系在基准气候与未来气候中保持不 变, 在探究物种多度对于未来气候变化的响应时, 也没有考虑物种之间的相互作用及其他影响物种分 布的环境因子。除气候因素外, 以上这些因素都会 影响物种的分布(Tessarolo et al., 2014)。本研究对于 当前 5 种树木多度的模拟是根据当前的实际多度所 建立起来的模型，已经包含了捕食、竞争以及人类 活动的影响，因此并不属于“基础生态位”的模拟, 但在未来气候条件下预测时, 只考虑了气候的变化 而没有考虑捕食、竞争等种间关系的变化以及人类 活动的干扰, 其结果则更接近于基础生态位的预测 (Stockwell \& Peters, 1999; Kearney \& Porter, 2004)。 此外, 虽然本文研究的 5 种树木大部分分布区均处 于中国境内, 境外分布区较小, 但这些数据的缺失 也限制了SDM模型的精度。

研究基于RF和基准气候、两个未来时期气候, 得到 5 种栋属植物多度的潜在分布和未来时期的多 度变化。内蒙古自治区东北部和黑龙江省北部地区 是本研究中 5 种栎属树木未来预测的多度集中减少 的地区, 该地区也是在2050年和2070年升温幅度最 大的区域。此外，陕西省中部也是在2050年预测下， 5 种栋属植物多度集中减小的地区。麻栎、葪栋、锐 齿葪柇和蒙古栋在未来预测中, 都有一半以上区域 的多度是减少的, 锐齿檞柇多度减少面积的比例达 到 $70 \%$ 以上。所以在未来气候变化下, 要加强对这 几个区域的监测与物种保护。

\section{参考文献}

Austin MP, Meyers JA (1996). Current approaches to modelling the environmental niche of eucalypts: Implication for management of forest biodiversity. Forest Ecology and Management, 85, 95-106.

Bellard C, Bertelsmeier C, Leadley P, Thuiller W, Courchamp F (2012). Impacts of climate change on the future of biodiversity. Ecology Letters, 15, 365-377.

Brito C, Crespo EG, Paulo OS (1999). Modelling wildlife distributions: Logistic multiple regression vs overlap analysis. Ecography, 22, 251-260.

Cao MC, Zhou GS, Weng ES (2005). Application and comparison of generalized models and classification and regression tree in simulating tree species distribution. Acta Ecologica Sinica, 25, 2031-2040. [曹铭昌, 周广胜, 翁恩 生 (2005). 广义模型及分类回归树在物种分布模拟中 的应用与比较. 生态学报, 25, 2031-2040.]
Editorial Board of the Chinese Vegetation Map, Chinese Academy of Sciences (2007). Vegetation Map of the People's Republic of China (1:1000 000). Geological Publishing House, Beijing. [中国科学院中国植被图编辑委 员会 (2007). 中华人民共和国植被图(1:100万). 地质出 版社, 北京.]

Chen IC, Hill JK, Ohlemuller R, Roy DB, Thomas CD (2011). Rapid range shifts of species associated with high levels of climate warming. Science, 333, 1024-1026.

Cutler DR, Edwards Jr TC, Beard KH, Cutler A, Hess KT, Gibson J, Lawler JJ (2007). Random forests for classification in ecology. Ecology, 88, 2783-2792.

Cutler K, Breiman L (2004). Random Forests. Machine Learning, 45, 157-176.

Domonkos P (2001). Temporal accumulations of extreme daily mean temperature anomalies. Theoretical and Applied Climatology, 68, 17-32.

Dubuis A, Pottier J, Rion V, Pellissier L, Theurillat JP, Guisan A (2011). Predicting spatial patterns of plant species richness: A comparison of direct macroecological and species stacking modelling approaches. Diversity and Distributions, 17, 1122-1131.

Ehrlén J, Morris WF (2015). Predicting changes in the distribution and abundance of species under environmental change. Ecology Letters, 18, 303-314.

Franklin J, Franklin JF, Franklin JD (2009). Mapping species distributions: Spatial inference and prediction. Quarterly Review of Biology, 36, 818.

Hao ZQ, Dai LM, He HS, Malandnoff DJ, Shao GF (2001). Potential response of major tree species to climate warming in Changbai Mountain, Northeast China. Chinese Journal of Applied Ecology, 12, 653-658. [郝占庆, 代力 民, 贺红士, Malandnoff DJ, 邵国凡 (2001). 气候变暖 对长白山主要树种的潜在影响. 应用生态学报, 12 , 653-658.]

Hastie T, Tibshirani R (1986). Generalized additive models. Statistical Science, 1, 297-310.

Hijmans RJ, Cameron SE, Parra JL, Jones PG, Jarvis A (2005). Very high resolution interpolated climate surfaces for global land areas. International Journal of Climatology, 25, 1965-1978.

Iverson LR, Prasad AM, Matthews SN, Peters M (2008). Estimating potential habitat for 134 eastern US tree species under six climate scenarios. Forest Ecology and Management, 254, 390-406.

Kaspari M (2001). Taxonomic level, trophic biology and the regulation of local abundance. Global Ecology and Biogeography, 10, 229-244.

Kearney M, Porter WP (2004). Mapping the fundamental niche: Physiology, climate, and the distribution of a nocturnal lizard. Ecology, 85, 3119-3131.

Kharin VV, Zwiers FW, Zhang X, Wehner M (2013). Changes in temperature and precipitation extremes in the CMIP5 ensemble. Climatic Change, 119, 345-357.

Li GQ, Liu CC, Liu YG, Yang J, Zhang XS, Guo K (2013). Advances in theoretical issues of species distribution 
models. Acta Ecologica Sinica, 33, 4827-4835. [李国庆, 刘长成, 刘玉国, 杨军, 张新时, 郭柯 (2013). 物种分 布模型的理论进展. 生态学报, 33, 4827-4835.]

Loehle C, LeBlanc D (1996). Model-based assessments of climate change effects on forests-A critical review. Ecological Modelling, 90, 1-31.

Nelder JA, Wedderburn RWM (1972). Generalized linear models. Journal of the Royal Statistical Society A, 135, $370-384$.

Parmesan C (2006). Ecological and evolutionary responses to recent climate change. Annual Review of Ecology, Evolution, and Systematics, 37, 637-669.

Peng YS, Chen L, Li JQ (2007). Study on numerical taxonomy of Quercus L. (Fagaceae) in China. Journal of Wuhan Botanical Research, 25, 149-157. [彭炎松, 陈丽, 李建强 (2007). 中国栋属植物的数量分类研究. 武汉植物学研 究, 25, 149-157.]

Picard R, Cook RD (1984). Cross-validation of regression models. Journal of the American Statistical Association, 79, 575-583.

Riahi K, Rao S, Krey V, Cho C, Chirkov V, Fischer G, Kindermann G, Nakicenovic N, Rafaj P (2011). RCP 8.5-A scenario of comparatively high greenhouse gas emissions. Climatic Change, 109, 33-57.

Rong YS, Tu QP (2001). Characteristics of evolution of potential evaporation in Tianjin region of China. Journal of Hydrodynamics, 16(3), 16-21.

Sainani KL (2013). Multivariate regression: The pitfalls of automated variable selection. $P M \& R, 5,791-794$.

Spano D, Cesaraccio C, Duce P, Snyder RL (1999). Phenological stages of natural species and their use as climate indicators. International Journal of Biometeorology, 42, 124-133.

Stockwell D, Peters D (1999). The GARP modelling system: Problems and solutions to automated spatial prediction. International Journal of Geographical Information Science, 13, 143-158.

Sturm M, Racine C, Tape K (2001). Increasing shrub abundance in the Arctic. Nature, 411, 546-547.

Syphard AD, Franklin J (2009). Differences in spatial predictions among species distribution modeling methods vary with species traits and environmental predictors. Ecography, 32, 907-918.

Tessarolo G, Rangel TF, Araújo MB, Hortal J (2014). Uncertainty associated with survey design in species distribution models. Diversity and Distributions, 20, 1258-1269.
Thuiller W (2003). Biomod-Optimizing predictions of species distributions and projecting potential future shifts under global change. Global Change Biology, 9, 1353-1362.

Thuiller W, Araújo MB, Lavorel S (2003). Generalized models vs. classification tree analysis: Predicting spatial distributions of plant species at different scales. Journal of Vegetation Science, 14, 669-680.

van Vuuren DP, Stehfest E, den Elzen MGJ, Kram T, van Vliet J, Deetman S, Isaac M, Klein Goldewijk K, Hof A, Mendoza Beltran A, Oostenrijk R, van Ruijven B (2011). RCP2.6: Exploring the possibility to keep global mean temperature increase below $2{ }^{\circ} \mathrm{C}$. Climatic Change, 109, 95-116.

Wang A, Goslee SC, Miller DA, Sanderson MA, Gonet JM (2017). Topographic variables improve climatic models of forage species abundance in the northeastern United States. Applied Vegetation Science, 20, 84-93.

Wang ZW, Ye JL, Yao TD (1998). Construction of mean annual temperature series for the last one hundred years in China. Quarterly Journal of Applied Meteorology, 9, 392-401. [王昭武, 叶瑾琳, 姚檀栋 (1998). 近百年中 国年气温序列的建立. 应用气象学报, 9, 392-401.]

$\mathrm{Xu} \mathrm{CH}, \mathrm{Xu}$ Y (2012). The projection of temperature and precipitation over China under RCP scenarios using a CMIP5 Multi-Model ensemble. Atmospheric \& Oceanic Science Letters, 5, 527-533.

Yan YJ, Li Y, Wang WJ, He JS, Yang RH, Wu HJ, Wang XL, Jiao L, Tang ZY, Yao YJ (2017). Range shifts in response to climate change of Ophiocordyceps sinensis, a fungus endemic to the Tibetan Plateau. Biological Conservation, 206, 143-150.

Young M, Carr MH (2015). Application of species distribution models to explain and predict the distribution, abundance and assemblage structure of nearshore temperate reef fishes. Diversity and Distributions, 21, 1428-1440.

Zhou GS, Zhang XS (1996). Study on Chinese climatevegetation relationship. Acta Phytoecologica Sinica, 20, 113-119. [周广胜, 张新时 (1996). 中国气候-植被关系 初探. 植物生态学报, 20, 113-119.]

Zhou ZK (1992). Origin, phylogeny and dispersal of Quercus from China. Acta Botanica Yunnanica, 14, 227-236. [周浙 昆 (1992). 中国栋属的起源演化及其扩散. 云南植物研 究, 14, 227-236.]

责任编委：王襄平 责任编辑：李 敏 实习编辑：赵 航

附录I 中国北方 5 种树种在 2050年RCP 2.6 和RCP 8.5下多度预测值

Supplement I Future distribution maps of abundance in the North China for five species under RCP 2.6 and RCP 8.5 scenarios in year 2050

http://www.plant-ecology.com/fileup/1005-264X/PDF/cjpe.2018.0249-S1.pdf

附录II 中国北方5种树种在2070年RCP 2.6 和RCP 8.5 下多度预测值

Supplement II Future distribution maps of abundance in the North China for five species under RCP 2.6 and RCP 8.5 scenarios in year 2070

http://www.plant-ecology.com/fileup/1005-264X/PDF/cjpe.2018.0249-S2.pdf

www.plant-ecology.com 
张雪晈, 高贤明, 吉成均, 康慕谊, 王仁卿, 岳明, 张峰, 唐志尧 (2019). 中国北方5种栋属 树木多度分布及其对未来气候变化的响应。植物生态学报, 43，774-782. DOI: 10.17521/cjpe.2018.0249

Zhang XJ, Gao XM, Ji CJ, Kang MY, Wang RQ, Yue M, Zhang F, Tang ZY (2019). Response of abundance distribution of five species of Quercus to climate change in northern China. Chinese Journal of Plant Ecology, 43, 774-782. DOI: 10.17521/cjpe.2018.0249

http://www.plant-ecology.com/CN/10.17521/cjpe.2018.0249

附录I 中国北方5种树种在2050年RCP 2.6和RCP 8.5下多度预测值

Supplement I Future distribution maps of abundance in the North China for five species under RCP 2.6 and RCP 8.5 scenarios in year 2050
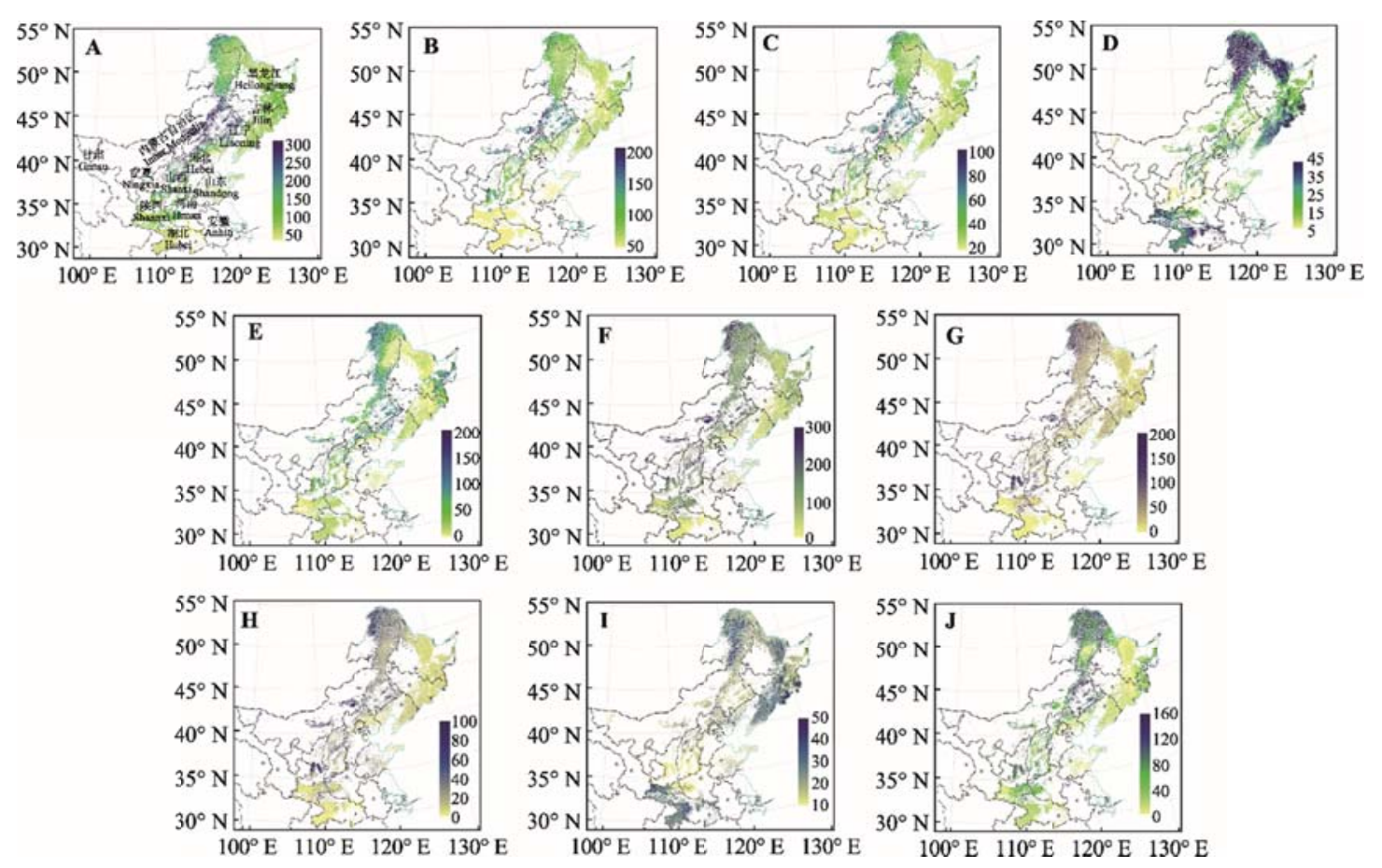

A, F, 栓皮柇。B, G, 麻柇。C, H, 葪柇。D, I, 锐齿触栎。E, J, 蒙古栎。

A, F, Quercus variabilis. B, G, Q. acutissima. C, H, Q. aliena. D, I, Q. aliena var. acuteserrata. E, J, Q. mongolica. 
张雪晈, 高贤明, 吉成均, 康慕谊, 王仁卿, 岳明, 张峰, 唐志尧 (2019). 中国北方5种栋属 树木多度分布及其对未来气候变化的响应。植物生态学报, 43，774-782. DOI: 10.17521/cjpe.2018.0249

Zhang XJ, Gao XM, Ji CJ, Kang MY, Wang RQ, Yue M, Zhang F, Tang ZY (2019). Response of abundance distribution of five species of Quercus to climate change in northern China. Chinese Journal of Plant Ecology, 43, 774-782. DOI: 10.17521/cjpe.2018.0249

http://www.plant-ecology.com/CN/10.17521/cjpe.2018.0249

附录II 中国北方5种树种在2070年RCP 2.6和RCP 8.5下多度预测值

Supplement II Future distribution maps of abundance in the North China for five species under RCP 2.6 and RCP 8.5 scenarios in year 2070
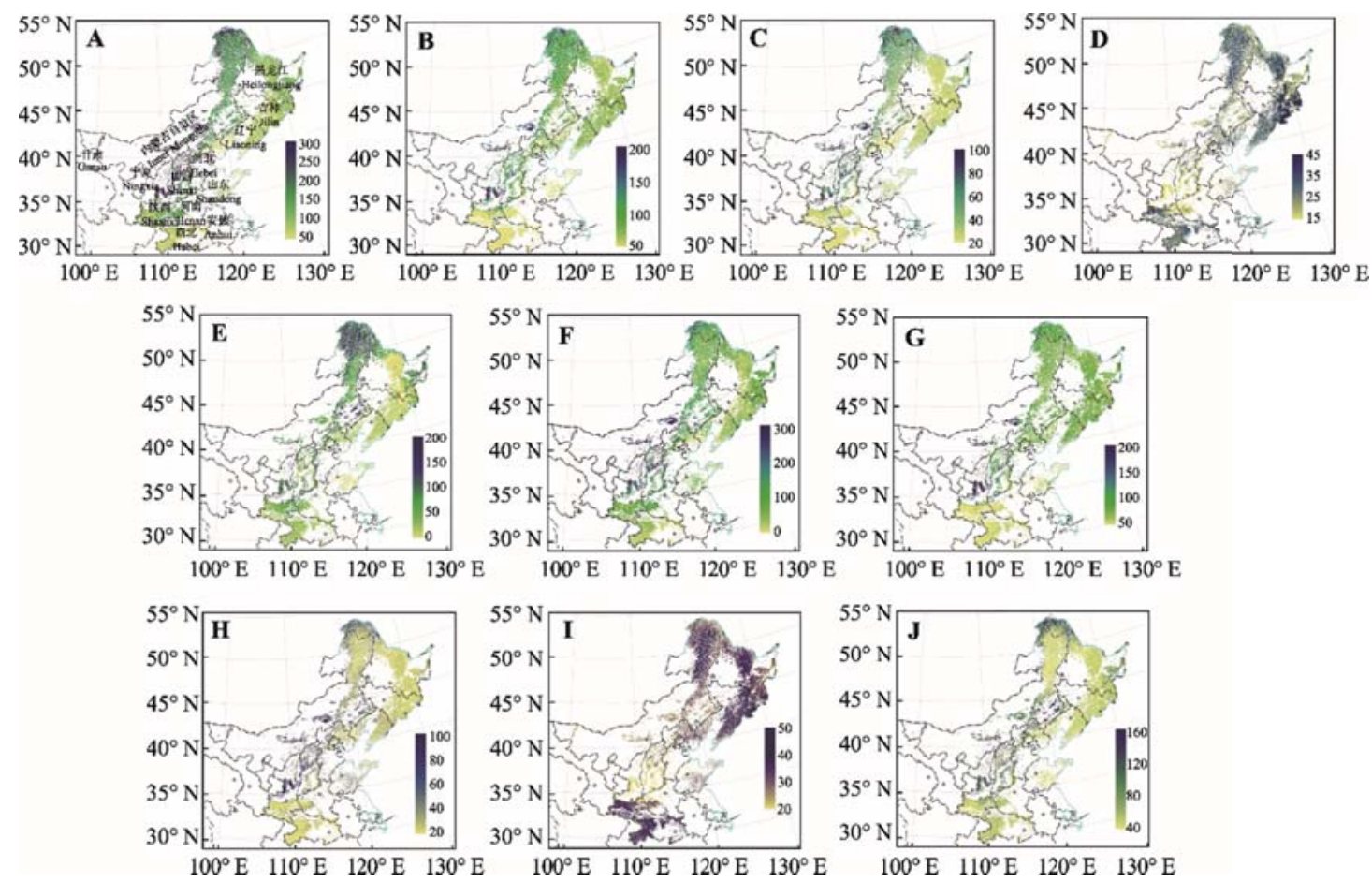

A, F, 栓皮栎。B, G, 麻栎。C, H, 檞柇。D, I, 锐齿檞栎。E, J, 蒙古栎。

A, F, Quercus variabilis. B, G, Q. acutissima. C, H, Q. aliena. D, I, Q. aliena var. acuteserrata. E, J, Q. mongolica. 